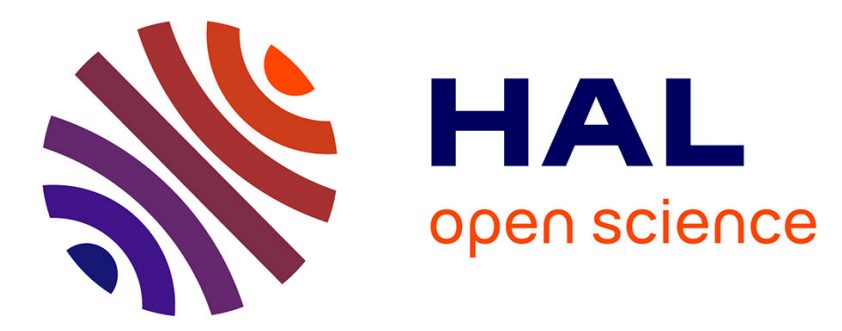

\title{
Random anisotropy effects in amorphous rare earth alloys (invited)
}

S. von Molnar, T. R. R Mcguire, R. J. Gambino, B. Barbara

\section{To cite this version:}

S. von Molnar, T. R. R Mcguire, R. J. Gambino, B. Barbara. Random anisotropy effects in amorphous rare earth alloys (invited). Journal of Applied Physics, 1982, 53 (11), pp.7666 - 7671. 10.1063/1.330178. hal-01660194

\section{HAL Id: hal-01660194 https://hal.science/hal-01660194}

Submitted on 10 Dec 2017

HAL is a multi-disciplinary open access archive for the deposit and dissemination of scientific research documents, whether they are published or not. The documents may come from teaching and research institutions in France or abroad, or from public or private research centers.
L'archive ouverte pluridisciplinaire $\mathbf{H A L}$, est destinée au dépôt et à la diffusion de documents scientifiques de niveau recherche, publiés ou non, émanant des établissements d'enseignement et de recherche français ou étrangers, des laboratoires publics ou privés. 


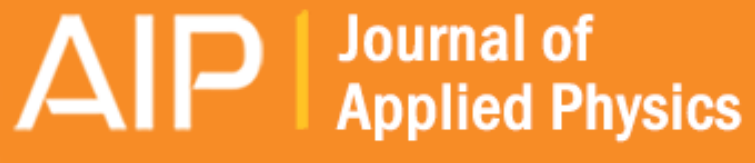

\section{Random anisotropy effects in amorphous rare earth alloys (invited)}

S. von Molnar, T. R. McGuire, R. J. Gambino, and B. Barbara

Citation: Journal of Applied Physics 53, 7666 (1982); doi: 10.1063/1.330178

View online: http://dx.doi.org/10.1063/1.330178

View Table of Contents: http://scitation.aip.org/content/aip/journal/jap/53/11?ver=pdfcov

Published by the AIP Publishing

\section{Articles you may be interested in}

The magnetic state and its macroscopic anisotropy in amorphous rareearth alloys (invited)

J. Appl. Phys. 67, 5769 (1990); 10.1063/1.345959

Magnetooptical measurement of anisotropy energy constants on amorphous rareearth transitionmetal alloys J. Appl. Phys. 67, 5328 (1990); 10.1063/1.344600

Random magnetism in amorphous rareearth alloys (invited)

J. Appl. Phys. 57, 3584 (1985); 10.1063/1.335014

A comparison of random anisotropy and spin glass properties in several amorphous rareearth alloys

J. Appl. Phys. 53, 2350 (1982); 10.1063/1.330816

Hall effect in rareearth-transitionmetal amorphous alloy films

J. Appl. Phys. 47, 3371 (1976); 10.1063/1.323098

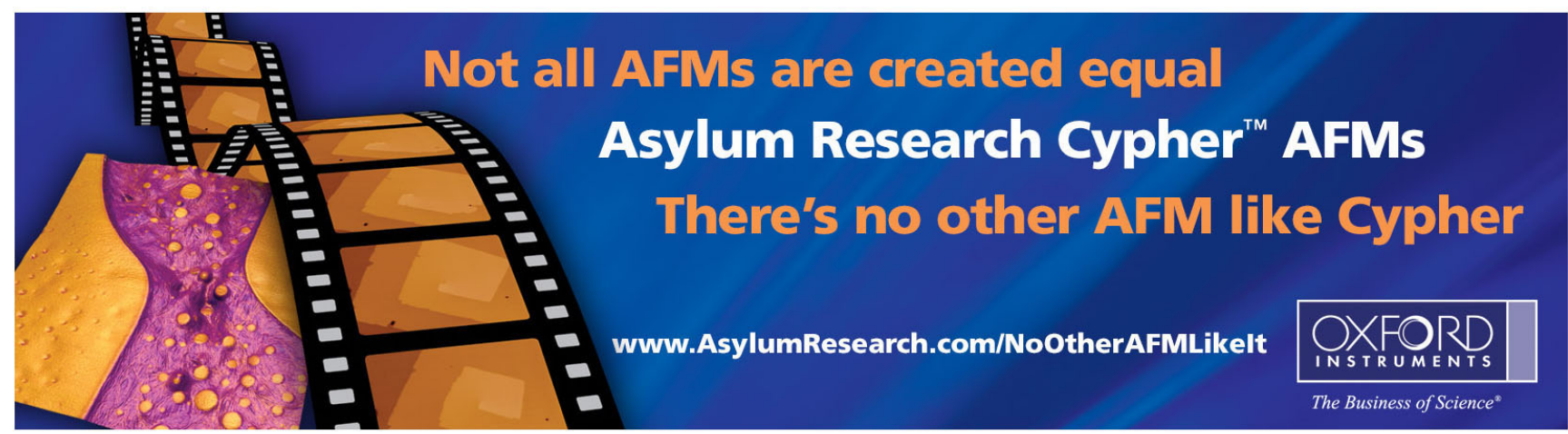




\title{
Random anisotropy effects in amorphous rare earth alloys
}

\author{
S. von Molnar, T. R. McGuire, and R. J. Gambino \\ (invited)
}

IBM Thomas J. Watson Research Center, Yorktown Heights, New York 10598

\section{B. Barbara}

\section{Laboratoire Louis Néel, CNRS, 166X, 38042 Grenoble, France}

We present specific heat and low field magnetic measurements on amorphous DyCu, DyAu, and DyNi alloy films. The magnetic contribution to the specific heat shows clearly that there are strong crystal fields effects in these systems. The highly anisotropic Dy alloys are compared to $G d$ alloys with similar exchange interactions but much weaker random anisotropy effects. Magnetic measurements on these two types of amorphous systems in the same field and temperature range show that they have characteristically different behavior. Using Arrot plots of our low field data we find that the $\mathrm{Gd}$ alloys all show evidence of spontaneous magnetization below $T_{C}$. The highly anisotropic Dy alloys do not develop a spontaneous moment at any temperature, a result in agreement with the theory of Aharony and Pytte.

PACS numbers: 75.10.Dg, 75.30.Gw, 75.40. $+\mathrm{t}$, 75.50.Kj INTRODUCTION

It has long been recognized $[1,2,3]$ that single ion anisotropy effects occur in many amorphous rare earth alloys. Theoretical considerations also suggest [3] that the local anisotropy axes are distributed randomly in space and recent Mössbauer measurements by Czjzek [4] indicate that local electric field gradients are consistent with this model. In order to explain the magnetic properties of such systems, Harris, Plischke and Zuckerman [2] introduced a Hamiltonian consisting of two terms, a Heisenberg exchange and a random uniaxial anisotropy,

$$
y=-\sum_{i \neq j} \mathrm{~J}_{i j} \vec{S}_{i} \cdot \vec{S}_{j}-D \sum_{i}\left(\vec{n}_{i} \vec{S}_{i}\right)^{2}, \quad D>0
$$

where $\mathrm{J}_{i j}$ is the exchange interaction, $\overrightarrow{\mathrm{S}}$ is the total angular momentum and $\vec{n}$ denotes the direction of the local anisotropy, $D$, at site $i$. A molecular field treatment of this model predicts a ferromagnetic state for positive average exchange interaction $\mathrm{J} \approx\left\langle\mathrm{J}_{\mathrm{ij}}\right\rangle$. Many of the magnetic properties of amorphous rare-earth alloys [1] have been interpreted as agreeing with this prediction. The same approach was used by Coey and von Molnar [5] to explain a large linear term in the low temperature specific heat of amorphous (a-) $\mathrm{Dy}_{.41} \mathrm{Cu}_{59}$. A subsequent study, however, of the magnetic phase diagram of a- $\mathrm{Dy}_{x} \mathrm{Cu}_{1-x}$ [6] was unable to establish the presence of long range magnetic order. In particular, Arrott plots of $M^{2}$ vs. $H / M$ (where $M$ is magnetization and $\mathrm{H}$ is the applied magnetic field), deduced from high field data failed to yield a definite value for the ferromagnetic transition temperature for most of the concentration range. Recently Coey, et al. [7] observed large Barkhausen jumps in low temperature hysteresis loops suggesting some ferromagnetic order in a-Dy-Cu. von Molnar et al. $[8]$, however, concluded from a detailed study of the low field dependence of the magnetization of a-Dy ${ }_{52} \mathrm{Cu}_{.48}$ that this highly anisotropic random magnet does not develop a moment at any temperature.

The present study, which is a review and extension of the earlier work $[5,6,7,8]$ was stimulated by the work of Aharony and Pytte [9] who pointed out that Arrott plots for random anisotropy systems have qualitatively different characteristics from those of ferromagnets and that these differences are most pronounced in the low field regime. These predictions are based on the assumption of positive $J$ only and random uniaxial anisotropy. In this investigation we present new specific heat data on a-Dy ${ }_{52} \mathrm{Cu}_{.48}, \mathrm{a}-\mathrm{Dy}{ }_{.54} \mathrm{Au}_{.46}$, and a-Dy ${ }_{32} \mathrm{Ni}_{68}$ which demonstrate the importance of single ion anisotropy and randomness on the thermal properties of Dy based alloys. We also present new measurements of the field dependent magnetic properties of amorphous $\mathrm{Dy}{ }_{54} \mathrm{Au}{ }_{46}$ and $\mathrm{Dy}{ }_{32} \mathrm{Ni}$ and compare them to their ferromagnetic counterparts a-GdAu $[10,11]$ and $a-G d N i$ $[12,13,14]$. These results, together with similar measurements on a-DY ${ }_{52} \mathrm{Cu}_{48}$ and $\mathrm{a}-\mathrm{Gd}_{52}-\mathrm{Ag}_{48}$ [8] (in which anisotropy is expected to be small because $\mathrm{Gd}$ is an $\mathrm{S}$ state ion) are discussed in the context of the Aharony-Pytte [9] predictions. We find that the amorphous Dy alloys, all of which are highly anisotropic magnets, do not develop a spontaneous moment at any temperature, in agreement with theory. It is, of course, possible to see a metastable remanence in large applied fields [7]. Various other details of the predictions are not evident in the data.

\section{EXPERIMENT}

a) Sample Preparation and Characterization

Our samples were prepared by two methods. Most of the films were either sputtered from alloy targets or co-evaporated onto glass. $\mathrm{Si}$, or $\mathrm{Al}_{2} \mathrm{O}_{3}$ substrates. The resulting films, which varied in thickness from $\sim 5000 \AA$ to $\sim 50,000 \AA$, have similar magnetic and thermal characteristics and thus do not appear to depend drastically on the method of preparation. The magnetic characteristics are preserved, even in a rapidly sputtered $160 \mu$ thick free standing sample of a-Dy$\mathrm{Cu}$ prepared for neutron diffraction studies. Preliminary specific heat data, however, do not agree in detail with those of the thinner samples. a-Gd-Ag films ( $6500 \AA$ ) could only be prepared by sputtering onto low temperature substrates. These films appear to be the least stable and tend to oxidize rapidly. The amorphous character of all films was verified by $X$-ray scattering, an upper limit on microcrystal size $(<16 \AA)$ being obtained with the use of the Scherrer formula [15]. The compositions of the films $( \pm 5$ wt. \%) were obtained by microprobe analysis. Sputtered films contained $\mathrm{Ar}$ and $\mathrm{O}_{2}$, whereas the evaporated films were cleaner.
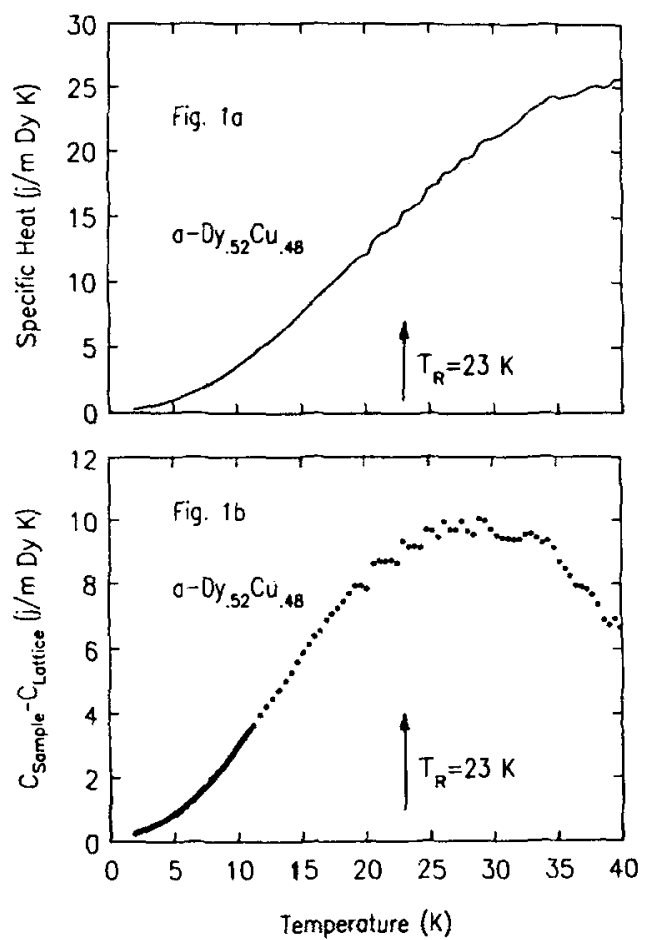

Fig. 1 Temperature dependence of the (a) total specific heat, $C$, and (b) specific heat without an estimated lattice term $\left(\theta_{D}=190 \mathrm{~K}\right)$ of a-Dy ${ }_{52} \mathrm{Cu}_{48} . \mathrm{T}_{\mathrm{R}}$, the magnetic critical temperature, is defined by a cusp in the low field d.c. susceptibility (see also Fig. 8) 
b) Specific Heat

The specific heat was measured by a thermal relaxation technique similar to that described by Forgan and Nedjat [16]. In the present experiments the amorphous films were deposited onto $\sim 75 \mu$ thick single crystal sapphire substrates and attached to a bolometerheater assembly with Apiezon $\mathrm{N}$ grease. In general, we measured both the sample- $\mathrm{Al}_{2} \mathrm{O}_{3}$ combination and the etched, clean $\mathrm{Al}_{2} \mathrm{O}_{3}$ blank. These data were then subtracted from one another to obtain the film specific heat. This procedure actually requires four independent measurements, since the bolometer and grease background is obtained prior to mounting the sample. The absolute error in the resulting specific heat of the thin film is estimated to be $\pm 12 \%$ (relative accuracies, of course, being much better). Errors in film thickness and estimated density may contribute $\sim 5 \%$ more to the values when normalized to moles of Dy.

The specific heat, $\mathrm{C}$, of $\mathrm{a}-\mathrm{Dy}_{1-x} \mathrm{Cu}_{\mathrm{x}}$ has been discussed in detail in Refs. 5 and 6 . Briefly, two experimental facts are noteworthy and exemplified by the new $\mathrm{Dy}_{{ }_{52}} \mathrm{Cu}_{48}$ data. To establish a temperature reference point, we define a temperature $T_{R}$, marked by a cusp in the low field d.c. susceptibility and, in the Dy alloys, the onset of irreversible effects in the magnetization. Further details will be given in the next section which discusses the magnetic data.

First of all, the magnetic contribution to the specific heat leads to an integrated entropy below $T_{R}$ which is close to $R \ln 2$ instead of RIn 16 anticipated for the Hund's rule coupled ground state value $\mathrm{J}=15 / 2$ of $\mathrm{Dy}^{3+}$ ion. This is clearly seen in Figures 1 and 2. Fig. $1 \mathrm{a}$ is the total specific heat of $\mathrm{Dy}_{52} \mathrm{Cu}_{48}$, while Fig. $1 \mathrm{~b}$ is the same data after an estimated lattice term (equivalent to a Debye temperature, $\theta_{\mathrm{D}}=190 \mathrm{~K}$ ) has been subtracted. We have assumed here that the specific heat of magnetic systems can be written as a sum of electronic, lattice and magnetic contributions, i.e.

$$
C=\gamma T+\beta T^{3}+C_{m}+\text { higher order terms }
$$

where $\gamma$ is an electronic term $=.5$ to $5 \times 10^{-3} \mathrm{j} /$ moleK, $\beta$ is the lowest order lattice term $=(1944 \times n) \div\left(\theta_{D}^{3}\right)$ and $n$ and is the number of atoms per formula unit. Figure 2 displays the entropy evolution as a function of temperature of the specific heat of Fig. $1 \mathrm{~b}$. Specifically,

$$
\text { Entropy }\left(T_{R}\right)=\int_{0}^{T} \mathrm{C} / T d T=R \ln (2 J+1) \text {. }
$$

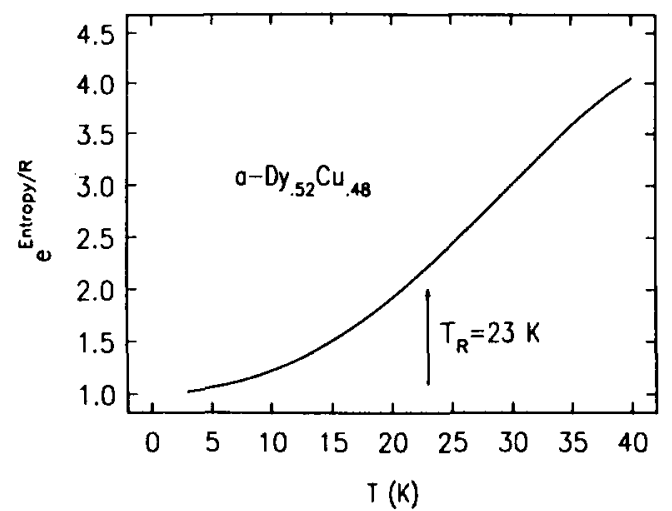

Fig. 2 Temperature variation of effective $(2 J+1)$ of a-Dy ${ }_{52} \mathrm{Cu}_{48}$ $(\theta=190 \mathrm{~K})$.

The ordinate of Fig. 2 thus represents the effective value of the argument $2 \mathrm{~J}+1$ as a function of temperature. Although no corrections have been made for any electronic contribution, $2 \mathrm{~J}+1$ is evidently closer to 2 than to 16 . This conclusion fortunately does not depend sensitively on the choice of $\theta_{D}$ - a parameter not very well known in these amorphous magnets. The fact that the entropy is $R \ln 2$ is a clear indication that crystal field effects are important in rare earth based amorphous alloys, particularly those with very large orbital angular momentum such as $D y^{3+}\left({ }^{6} \mathrm{H}_{15 / 2}\right)$. In fact $R \ln 2$ is the entropy expected for the Zeeman splitting of a single Kramers doublet. The remainder of the entropy will be developed upon population of the other crystal field levels, at higher temperatures. Similar results have been obtained for all of the Dy alloys we have studied and the results of both the total specific heat and magnetic entropy are given in Figs. 3, $4,5,6$.

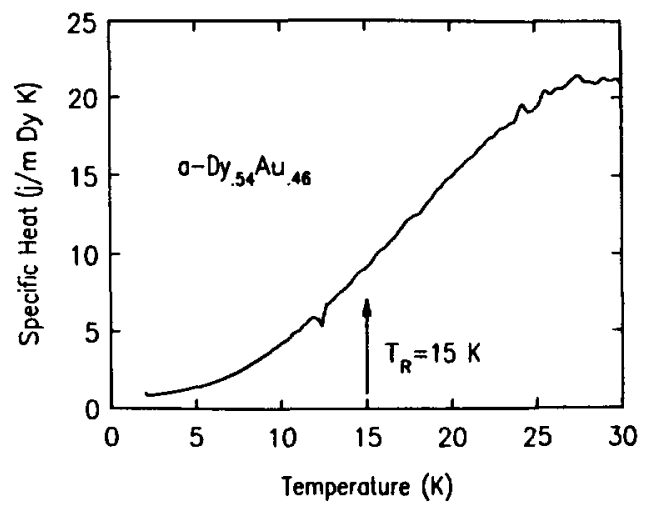

Fig. 3 Temperature dependence of the total specific heat, $C$, of a-Dy. ${ }_{.54} \mathrm{Au}_{.46}$.

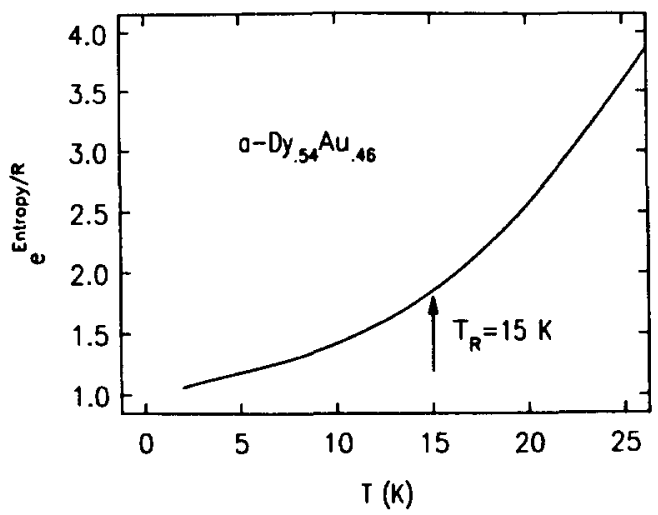

Fig. 4 Temperature variation of effective $(2 \mathrm{~J}+1)$ of a-Dy ${ }_{.54} \mathrm{Au}_{.46}$ $(\theta=200 \mathrm{~K})$

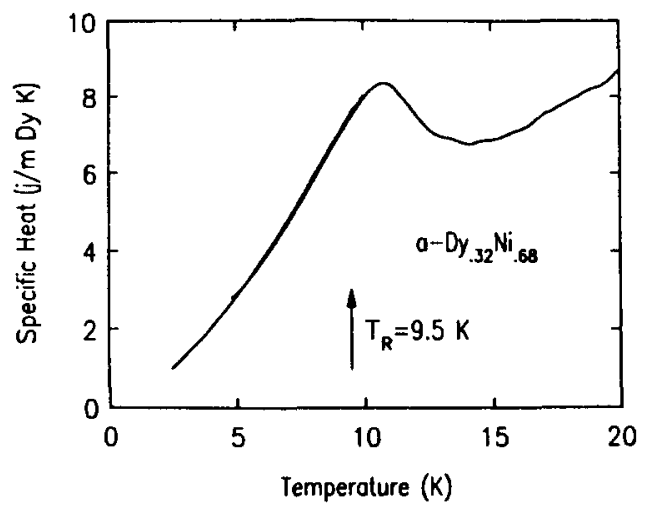

Fig. 5 Temperature dependence of the total specific heat, $\mathrm{C}$, of a-Dy ${ }_{.32} \mathrm{Ni} .68$.

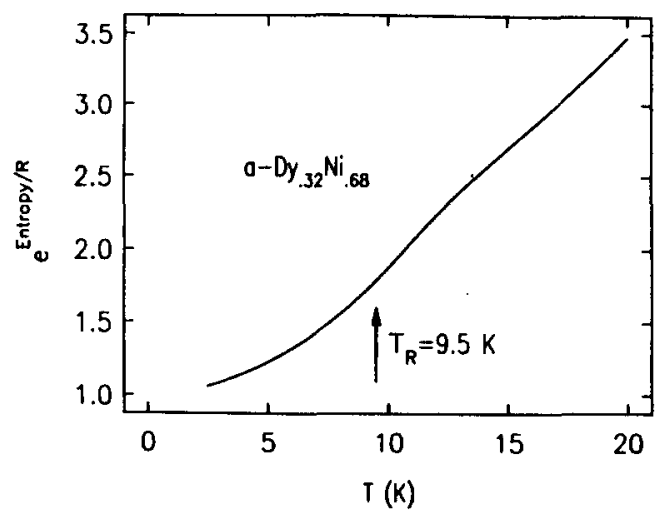

Fig. 6 Temperature variation of effective $(2 \mathrm{~J}+1)$ of $\mathrm{a}-\mathrm{Dy}_{{ }_{32}} \mathrm{Ni}_{6}{ }_{68}$ $(\theta=210 \mathrm{~K})$. 
The second noteworthy result is contained in Fig. 7 where we plot $\mathrm{C} / \mathrm{T}$ vs. $\mathrm{T}^{2}$ for $\mathrm{Dy}_{.52} \mathrm{Cu}_{48}$. An extrapolation to $\mathrm{T}^{2} \rightarrow 0$ yields a value for the coefficient of the linear term $\gamma=125 \times 10^{-3} \mathrm{j} /$ moleDy $\mathrm{K}$, approximately two orders of magnitude greater than any normal electronic contribution.

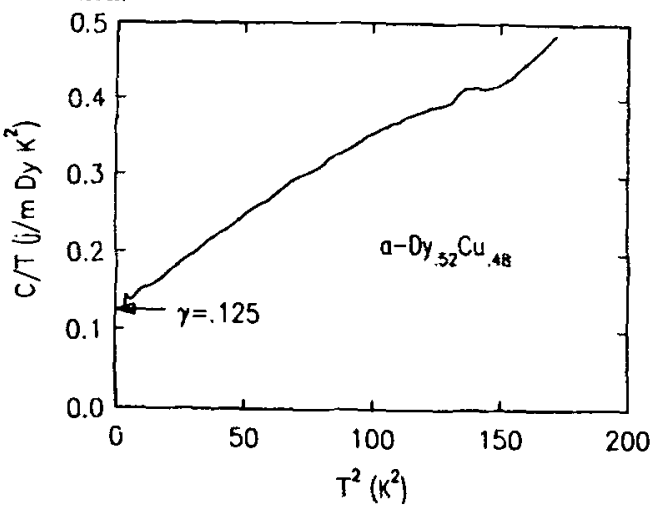

Fig. 7 Low temperature total specific heat of a- $\mathrm{Dy}{ }_{.52} \mathrm{Cu}_{48}$ plotted as $\mathrm{C} / \mathrm{T}$ vs. $\mathrm{T}^{2}$ to display the large linear term, $\gamma \approx 125 \times 10^{-3^{8}} \mathrm{j} / \mathrm{mole}$ Dy

A natural explanation of this term follows if one assumes that the system is completely random and that $\mathrm{D} / \mathrm{J}>1[5]$. Unde these circumstances (see Eq. 1) the (uniaxial) crystal field splits the $J=15 / 2$ ground state into 8 doublets separated from one another by energies proportional to $D$. At low temperatures we may ignore all but the lowest of these doublets $\left(S_{7}= \pm 15 / 2\right)$ and consider the effect on one Dy spin along its $\mathrm{z}$ anisotropy axis due to all the other spins. If one assumes that the effective molecular field acting on $S_{z}$ is of constant magnitude $\mathrm{H}_{\mathrm{m}}$ in direction $\mathrm{z}^{\prime}$, the Zeeman energy at site $i$ will be $\boldsymbol{g}_{\mathrm{i}}= \pm \mathrm{g} \mu_{\beta} \mathrm{H}_{\mathrm{m}}^{\mathrm{i}} \cdot \mathrm{S}_{\mathrm{z}}^{\mathrm{i}}=\mathrm{A} \cos \theta_{\mathrm{i}}$, from which we can easily calculate the specific heat if the distribution of $\theta_{i}$, the angle between the molecular field and anisotropy axes are known. In particular

$$
\mathrm{C}=\frac{\partial \mathrm{U}}{\partial \mathrm{T}}=\frac{\partial}{\partial \mathrm{T}} \int \mathrm{d} \Omega \mathrm{P}(\Omega)\left\{\frac{-\mathscr{E} \mathrm{e}^{+\varepsilon / \mathrm{kT}}+\mathbb{\mathrm { e } ^ { - \varepsilon / k T }}}{\mathrm{e}^{-\varepsilon / \mathrm{kT}}+\mathrm{e}^{+\varepsilon / \mathrm{kT}}}\right\}
$$

where $d \Omega=\sin \theta \mathrm{d} \theta \mathrm{d} \phi$ and $\mathrm{P}(\Omega)=$ probability of finding any solid angle, $\Omega$. For a completely random system, $P(\Omega)=1$ and the integral of Eq. 1 may be evaluated approximately, yielding that

$$
\mathrm{C}=\frac{\pi^{2} \mathrm{Nk}^{2} \mathrm{~T}}{12 \mathrm{~g} \mu_{\beta} \mathrm{H}_{\mathrm{m}} \mathrm{S}_{\mathrm{z}}}
$$

A more complete derivation has been given by Korenblit and Shender [17]. Two comments need be made about Eq. 5. The coefficient of the linear term can be evaluated to yield $\mathrm{H}_{\mathrm{m}}$ from which a rough estimate of $T_{R}$ may be obtained. [5] For the Dy $\mathrm{Cu}_{x}$ system the agreement with the measured $T_{R}$ is satisfactory, and lends credence to the formula, Eq. 5. Most importantly, the linearity is a direct result of assuming that the anisotropy axes are uncorrelated, i.e. that they are random. If this were not the case, the density of Zeeman split states would not be constant in energy between $\pm \mathrm{g} \mu_{\mathrm{B}} \mathrm{H}_{\mathrm{m}} \mathrm{S}_{7}$ resulting in a gap or decrease in state density. This, in turn, would produce nonlinear effects at low temperatures. The fact that such deviations are not observed in $\mathrm{Dy}_{52} \mathrm{Cl}_{48}$ down to $2 \mathrm{~K}$ supports our supposition of random uniaxial anisotropy in this alloy.

We have observed nonlinear effects in other alloys [5] notably in preliminary measurements of a $160 \mu$ thick freestanding a-DyCu platelet and tentatively attribute these to nonrandom distribution of axes. Furthermore, the extrapolation to separate the linear from othe terms in Eq. 2 is often nontrivial. A case in point is a-DyAu in which a low temperature contribution of unknown origin obscures the linear term below $4 \mathrm{~K}$, or a-DyNi, where we were unable to perform measurements to low enough temperatures below $T_{R} \simeq 11 \mathrm{~K}$ to extract the linear term.

\section{c) Magnetic Properties}

Magnetic measurements were made using a SHE SQUID magnetometer capable of operating in applied magnetic fields between $\sim 1$ and 50,000 oe and temperatures between 4.3 and $\sim 300 \mathrm{~K}$. Above $60 \mathrm{~K}$, temperature equilibrium requires times in excess of 30 minutes per point and accounts for the lower density of data for a-Gd-Ag near $\mathrm{T}_{\mathrm{R}} \sim 80 \mathrm{~K}$. Approximate relative accuracies are as follows: $\Delta \mathrm{M} / \mathrm{M} \sim 10^{-3} ; \quad \Delta \mathrm{T} \sim \pm 0.2 \mathrm{~K}(\mathrm{~T} \leq 70 \mathrm{~K}) ; \quad \Delta \mathrm{T} \sim \pm 1 \mathrm{~K}(\mathrm{~T}>70 \mathrm{~K})$ The absolute accuracy of the temperature below $70 \mathrm{~K}$, where most measurements were made, is approximately $\pm 1 \mathrm{~K}$. In all cases the applied field, $\mathrm{H}$ was parallel to the plane of the film; its absolute accuracy increasing from $\sim \pm 5 \%$ to $\sim \pm 2 \%$ with increasing field. Since much of the analysis involves data with applied fields of $200 \mathrm{Oe}$ and less, we followed a calibration procedure for obtaining $\mathrm{H}$ from the measured magnetization at a high enough temperature where $\mathbf{M} \propto \mathrm{H}$. In addition, most of the fields below 50 Oe were checked with the diamagnetic susceptibility of a superconducting $\mathbf{P b}$ sphere.

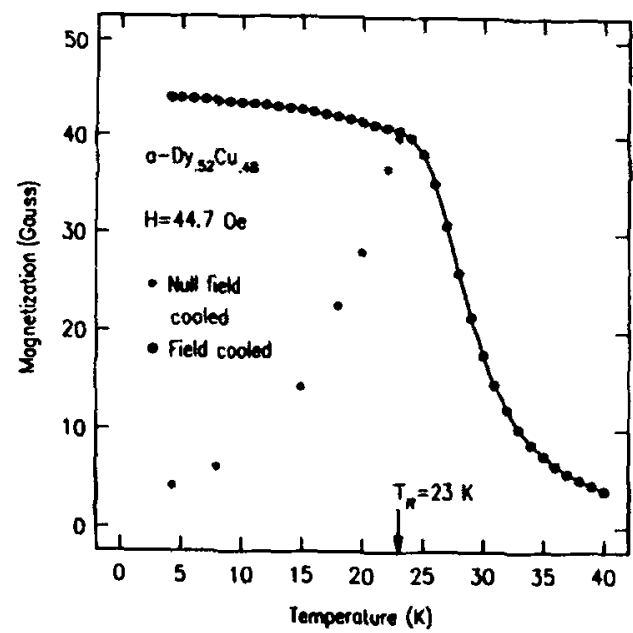

Fig. 8 Magnetization vs, temperature. The open circles represent data obtained after cooling the sample to $4.2 \mathrm{~K}$, applying the field ( $44.7 \mathrm{Oe})$ and measuring the magnetization during the warming cycle. Above the peak at $T_{R}=23 \mathrm{~K}$, the magnetization is reversible. The solid dots below $T_{R}$ are the magnetization upon cooling in the field.

Typical data for a-Dy ${ }_{52} \mathrm{Cu}_{.48}$, are shown in Fig. 8. The samples show a sharp peak at $T_{R}=23 \mathrm{~K}$ in the zero field cooled magnetization $(\sim 10$ minutes/point $)$. The open circles are measured with increasing temperature after the field has been increased from null to $44.7 \mathrm{Oe}$. The upper curve (solid line) is the measured magnetization when the sample is cooled in the measuring field of $44.7 \mathrm{Oe}$ This magnetization is time independent throughout the entire temperature range. We will consider only these field cooled curves in our discussion of the data. In particular, our concern is with the behavior near $T_{R}$, where we have checked that the magnetization is the same during heating and cooling within our experimental accuracy $\Delta M / M \sim 10^{-5}$ and time delays between points of order 10 minutes.

A principal purpose of this paper is to compare our results (for two extreme cases, $\mathrm{D} / \mathrm{J}>1$ and $\mathrm{D} / \mathrm{J}<<1$ ) to the predictions of Aharony and Pytte. [9] It will be convenient therefore to review the salient features of that theory. In order to obtain the leading term in the magnetic equation of state for a system described by Eq. 1, these authors assumed the following: (a) positive J only; (b) random anisotropy, i.e. equal probability for $\vec{n}$ in all directions; (c) fluctuation effects (which play an important role in determining critical exponents near the phase transition in normal ferromagnets) are neglected. Within this framework Aharony and Pytte [9] predict that a random anisotropy system will undergo a second order phase transition at temperature, $T_{R}$, to a very unusual, new magnetic state: an infinite susceptibility for $T \leq T_{R}$, but no spontaneous magnetization at any temperature. In addition the following values for the exponent, $\delta$, describing the field dependence of magnetization $\left(H \propto M^{\delta}\right)$ are expected for a three dimensional system: (a) $\delta=5$ for $T<T_{R} \delta=7 / 3$ for $\mathrm{T}=\mathrm{T}_{\mathrm{R}}$. Of course, for $\mathrm{T} \gg \mathrm{T}_{\mathrm{R}}, \delta$ must reach the classical value $\delta=1$.

In our discussion of the specific heat results we demonstrated the importance of random single ion anisotropy in all of the Dy alloys. In particular, a-Dy-Cu, satisfies the randomness condition (b), above. Condition (a), positive J only, is more difficult to establish. Although the paramagnetic Curie temperature, $\theta$, which reflects the sum of the exchange interactions, is positive in all of the alloys studied (see Table 1), the existence of a distribution in $\mathrm{J}$ having some negative contribution cannot be ruled out. We have therefore studied, in addition to a-Dy ${ }_{52}-\mathrm{Cu}_{4}{ }_{4},[8]$ the alloys a-Dy ${ }_{54} \mathrm{Au}_{46}$ and $\mathrm{a}-\mathrm{Dy}{ }_{32} \mathrm{Ni}_{68}$. Corresponding $\mathrm{Gd}-\mathrm{Au}$ and $\mathrm{Gd}-\mathrm{Ni}$ alloys (ground state configuration ${ }^{8} \mathrm{~S}_{7 / 2}$ ), in which anisotropy effects are small, order maznetically. Poon and Durand [10] have shown that $\mathbf{a}-\mathrm{Gd}_{80} \mathrm{Au}_{20}$ is a well behaved ferromag-

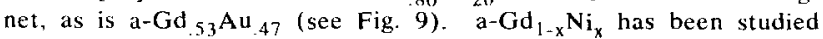
extensively $(12,13,14]$. These materials are found to be soft ferromag- 
nets, albeit with a small induced antiparallel Ni moment for $x>0.7$. We therefore expect that condition (a) will be satisfied in the Au and $\mathrm{Ni}$ alloys containing Dy. Of course, $\mathrm{J}$ will be larger in the Gd alloys in accordance with the well known de Gennes factor.

To complete our study we have measured the magnetization of two other amorphous $\mathrm{Gd}$ alloys $(\mathrm{D} / \mathrm{J}<<1)$. The first of these, $\mathrm{Gd}_{52} \mathrm{Ag}_{48}$, was chosen because earlier work by Boucher [18] in high fields indicated that low field magnetization measurements might show the behavior predicted by Aharony and Pytte [9]. The other, a-Gd ${ }_{37} \mathrm{Al}_{63}$, is a well known spin glass. $[19,20]$ Salient properties of the alloys under discussion are given in Table $\mathrm{I}$.

\section{Table I}

\begin{tabular}{|c|c|c|c|c|c|c|c|c|c|c|}
\hline $\begin{array}{l}\text { Alloy } \\
\text { at } \%\end{array}$ & $\begin{array}{c}\mathbf{M}_{\mathrm{o}} \\
\text { (Gauss) }\end{array}$ & $\begin{array}{c}\mathrm{M} \\
\text { (Gauss) }\end{array}$ & $M_{s /} M_{0}$ & $\begin{array}{l}T_{R} \\
(K)\end{array}$ & $\begin{array}{l}T_{c} \\
(K)\end{array}$ & $\begin{array}{c}\theta \\
(\mathrm{K})\end{array}$ & $\begin{array}{c}\mathrm{J} \\
(\mathrm{kOe})\end{array}$ & $\begin{array}{l}\mathrm{H}_{\mathrm{A}}(\mathrm{D}) \\
(\mathrm{kOe})\end{array}$ & $\begin{array}{c}K_{u} \\
(e r g s / c c)\end{array}$ & $\mathrm{D} / \mathrm{J}$ \\
\hline Dy ${ }_{54} \mathrm{Au}_{46}$ & 2015 & 860 & 0 & 15 & & 17 & 61 & 173 & $9.6 \times 10^{7}$ & 2.8 \\
\hline $\mathrm{Dy}_{.52} \mathrm{Cu}_{48}$ & 2170 & 1300 & 0 & 23 & & 30 & 108 & 13 & 10.4 & 1.5 \\
\hline $\mathrm{Dy}_{.32} \mathrm{Ni}_{68}$ & 1674 & 1110 & 0 & 9.5 & & 12 & 43 & 221 & 7.6 & 5.1 \\
\hline $\mathrm{Gd}_{52} \mathrm{Au}_{48}$ & 1337 & 1365 & $\sim 1$ & & 70 & & 350 & & & \\
\hline $\mathrm{Gd}_{52} \mathrm{Ag}_{4 \mathrm{~K}}$ & 1325 & 875 & .18 & & 83 & & 415 & & & \\
\hline $\mathrm{Gd}_{23} \mathrm{Ni}_{77}$ & 926 & 818 & $\sim .9$ & & 36 & & 175 & & & \\
\hline
\end{tabular}

$M_{0} \quad$ calculated for all spins parallel.

$\mathrm{M}$ measured at $5^{\circ} \mathrm{K}$ in $40 \mathrm{kG}$.

$\mathrm{M}_{\mathrm{S}} / \mathrm{M}_{\mathrm{O}} \quad$ spontaneous moment ratio where $\mathrm{M}_{\mathrm{s}}$ is determined from extrapolations of $\log \mathrm{M}$ vs. $\log \mathbf{H}$ plots evaluated at $\mathrm{H}$. NM (see text).

$\mathrm{T}_{\mathrm{R}}$ random anisotropy transition based on peak in field cooled measurement (see text).

J Exchange field calculated from $\theta$ or $\mathrm{T}_{\mathrm{c}}$.

$\mathrm{H}_{\mathrm{A}}$ anisotropy field estimated from projection of $\mathbf{M}-\mathrm{H}$ slope at $5^{\circ} \mathrm{K}$ to saturation $\left(\mathrm{M}_{\mathrm{o}}\right)$ which is used as a measure of $\mathrm{D}$, the local anisotropy

$\mathrm{K}_{\mathrm{u}} \quad$ calculated from the area associated with the $\mathrm{M}-\mathrm{H}$ plot at $5^{\circ} \mathrm{K}$.

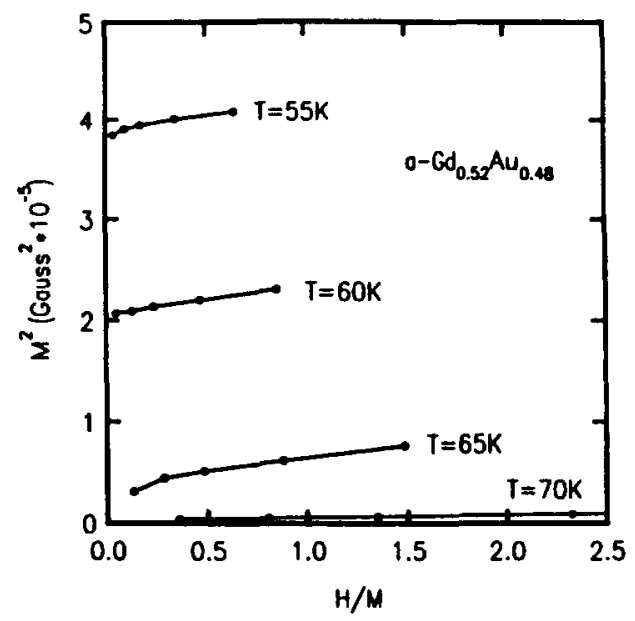

Fig. 9 Arrott $\left(\mathrm{M}^{2}\right.$ vs. $\left.\mathrm{H} / \mathrm{M}\right)$ plots of $\mathrm{a}-\mathrm{Gd}_{.52} \mathrm{Au}_{.48}$ for applied fields between 23 and $440 \mathrm{Oe}$.

To demonstrate the drastic changes effected by the random anisotropy, Aharony and Pytte [9] compared Arrott plots, i.e. $\mathbf{M}^{2}$ vs $H / M$ for the two cases $D / J \neq 0$ and $D / J=0$. We follow the same procedure to display the magnetic results. For the present it is only necessary to recognize that intercepts along the abscissa define the limiting inverse susceptibility, $\chi^{-1}(T)$, and that intercepts along the positive ordinate represent the square of the spontaneous magnetization. In a normal ferromagnet, the isotherm which goes through the origin defines the thermodynamic transition temperature to long range order. [21] For $\mathrm{Gd}_{52} \mathrm{Au}_{48}$ (see Fig. 9) this temperature is clearly between 65 and $70 \mathrm{~K}$. In the case of the Dy alloys (Figs. 10,11 and 12) we make the following observations: (a) there is no spontaneous magnetization down to $4.2 \mathrm{~K}$; (b) the susceptibility remains finite for all $T$, i.e. no isotherm reaches 0 on the abscissa; (c) there appear to be two types of behavior near the origin of the curves. The Ni alloy plot has negative curvature below $T_{R}$ (see Fig. 10b), whereas the curvature of both the $\mathrm{Au}$ and $\mathrm{Cu}$ curves (Figs. 11 and 12) is positive; (d) as $\mathrm{H} / \mathrm{M}$ increases the curves become similar, the negative curvature becoming less pronounced and in high fields (see for example insert, Fig. 12) the magnitude of the negative curvature changes as a function of temperature. This last observation explains why high field extrapolations, which give a spontaneous moment, are misleading and indicates a drastic change in the field dependence of $M$ near $T_{R}$.
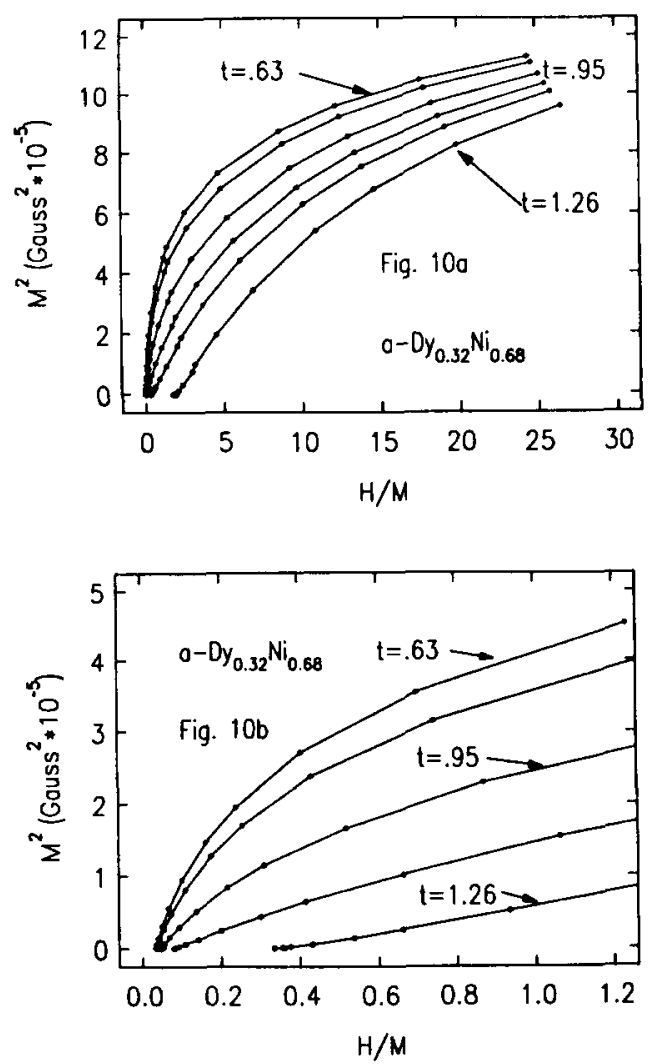

Fig. 10 Arrott ( $\mathrm{M}^{2}$ vs. $\left.\mathrm{H} / \mathrm{M}\right)$ plots of a-Dy ${ }_{32} \mathrm{Ni}{ }_{68}$ for applied fields up to (a) $26 \mathrm{kOe}$ (b) $800 \mathrm{Oe}$. The reduced temperature $\mathrm{t}=\mathrm{T} / \mathrm{T}_{\mathrm{R}}=\mathrm{T} / 9.5 \mathrm{~K}$

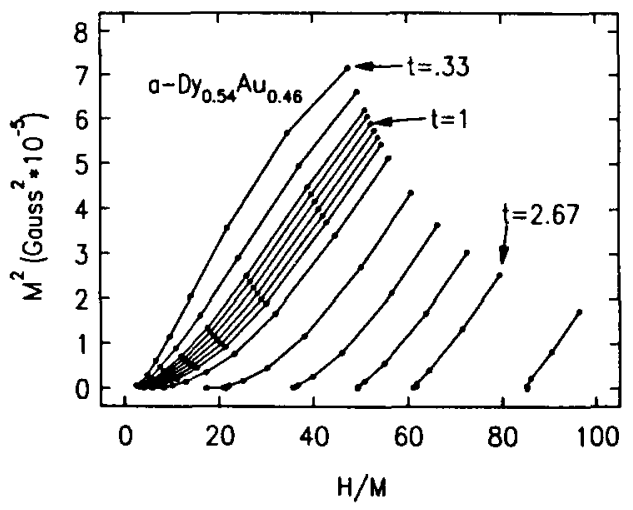

Fig. 11 Arrott $\left(\mathrm{M}^{2}\right.$ vs. $\left.\mathrm{H} / \mathrm{M}\right)$ plots of $\mathrm{a}-\mathrm{Dy}{ }_{54} \mathrm{Au}{ }_{46}$ for applied fields up to $40 \mathrm{kOe}$. The reduced temperature $t=\mathrm{T} / \mathrm{T}_{\mathrm{R}}=\mathrm{T} / 15 \mathrm{~K}$.

Figure 13 is a similar Arrott plot for a-Gd ${ }_{52} \mathrm{Ag}_{48}$. In comparison with the $\mathrm{Dy}-\mathrm{Cu}$ results, we see that: (a) there is no clear evidence of spontaneous magnetization below $T_{R}$, although, as we shall discuss, this conclusion depends critically on the magnitude of any possible extremely small demagnetizing field; (b) $x^{-P} \rightarrow 0$, i.e., the susceptibility appears to become infinite, for $T \leq T_{R}$; (c) the sign of the curvature (negative) remains the same with increasing field below $\mathbf{T}_{\mathbf{R}}$. 


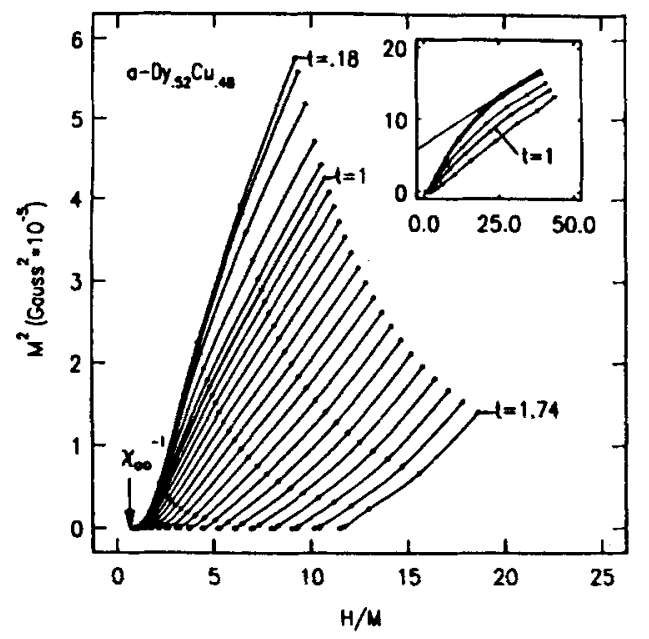

Fig. 12 Arrott ( $\mathrm{M}^{2}$ vs. $\mathrm{H} / \mathrm{M}$ ) plots of a-Dy ${ }_{52} \mathrm{Cu}_{48}$ for applied fields up to $7 \mathrm{kOe}$. The insert is the same plot extended to $50 \mathrm{kOe}$. The dashed line represents incorrect extrapolation from high fields giving an apparent moment. The reduced temperature $t=T / T_{R}=T / 23 \mathrm{~K}$.

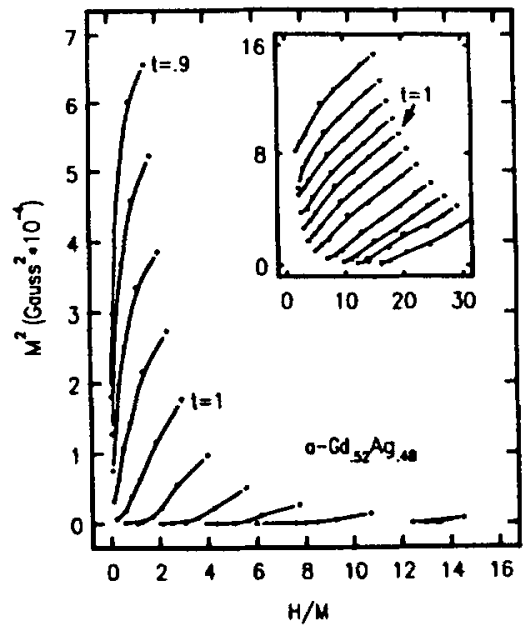

Fig. 13 Arrott ( $\mathrm{M}^{2}$ vs. $\left.\mathrm{H} / \mathrm{M}\right)$ plots of $\mathrm{a}-\mathrm{Gd}{ }_{.52} \mathrm{Ag}{ }_{48}$ for applied fields up to $400 \mathrm{Oe}$. The insert is the same plot for intermediate fields $500 \mathrm{Oe} \leq \mathrm{H} \leq 6000 \mathrm{Oe}$ obtained with a Faraday Balance. The reduced temperature $t=T / T_{R}=T / 83 K$.

We shall focus the remainder of the detailed discussion concerning the Dy alloys on a-Dy ${ }_{52} \mathrm{Cu}_{48}$, for which the data is most complete. Referring to the results in Fig. 12, the most striking agreement with the theory is the obvious absence of a spontaneous magnetization at all temperatures. However, contrary to theory, the reciprocal susceptibility, $\chi_{0}^{-1}(\mathrm{~T})$, remains finite at all temperatures. Furthermore, it is nearly temperature independent below $T_{R}$ :

$$
\begin{aligned}
& x_{0}^{-1}(23 \mathrm{~K})=x_{0}^{-1}\left(\mathrm{~T}_{\mathrm{R}}\right)=0.84, \\
& x_{0}^{-1}(4.2 \mathrm{~K})=x_{0}^{-1}\left(0.18 \mathrm{~T}_{\mathrm{R}}\right)=0.81
\end{aligned}
$$

The low temperature reciprocal susceptibility $x_{0}^{-1}\left(0.18 T_{R}\right)=x_{o o}^{-1}$ is not limited by geometric demagnetizing effects which are at least two orders of magnitude smaller, but instead seems to be associated with a homogeneous freezing at $T_{R}$. To see if this freezing, at $T_{R}$, is related to a phase transition we include higher order terms in the expansion of the magnetization by the simple device of translating the $M^{2}$ axis in Fig. 12 by $-x_{00}^{-1}$. Thus, for $T<T_{R}$ and $H \leqslant 1$ kOe we have

$$
\mathbf{H}=\chi_{\mathrm{oo}}^{-1} \mathrm{M}+\chi_{1}^{-1}(\mathrm{~T}) \mathbf{M}^{\delta}
$$

where $\delta \simeq 2$. More accurate values for exponents are found by plotting $\log \mathbf{H}_{\text {eff }}=\mathbf{H}-\chi_{00}^{-1} \mathbf{M}$ vs. $\log \mathbf{M}$ at each temperature and determining $\delta$ from the slope. These results are shown in Fig. 14 (solid points) together with the least square error over the limited range, $1 \mathrm{Oe}<\mathrm{H}_{\text {eff }} \leq 1000 \mathrm{Oe}$. In summary: $\delta=1 \pm .05$ for $\mathrm{T}>1.5 \mathrm{~T}_{\mathrm{R}}$; $\delta=2.0 \pm .1$ at $\mathrm{T}_{\mathrm{R}}$; and $\delta=2.1 \pm .03$ for $\mathrm{T}=.18 \mathrm{~T}_{\mathrm{R}}$. A similar analy- sis for a-Dy ${ }_{32} \mathrm{Ni}_{68}$ gives: $\delta=1 \pm .05$ for $\mathrm{T}>>\mathrm{T}_{\mathrm{R}} ; \delta>4$ for $\mathrm{T} \ll \mathrm{T}_{\mathrm{R}}$. It was not possible to define a linear region for $\log \mathrm{M}$ vs. $\log \mathrm{H}$ at $\mathrm{T}=\mathrm{T}_{\mathrm{R}}=9.5 \mathrm{~K}$. For a-Gd ${ }_{52} \mathrm{Ag}_{\text {.4x }}$ (Fig. 14, open circles) the same analysis yields the following: $\delta=1 \pm .05$ for $T>1.1 \mathrm{~T}_{R}$ $\delta=2.5 \pm .1$ at $\mathrm{T}_{\mathrm{R}}$ and $6.3 \pm .2$ for $\mathrm{T}=.9 \mathrm{~T}_{\mathrm{R}}$. Here the exponent $\delta$ was determined directly from $\log \mathrm{M}$ vs. $\log \mathrm{H}$ plots. No linear term of the form $\chi_{00}^{-1} M$ appears because, as is apparent from Fig. 13, H/M approaches 0 ( $x$ diverges) at $T_{R}=83 \mathrm{~K}$. In fact, the Arrott plot for a-Gd ${ }_{52} \mathrm{Ag}_{.48}$ (Fig. 13) appears to have the shape predicted by Aharony and Pytte. [9] However $\delta$ continued to increase below $\mathrm{T}_{\mathrm{R}}$.

The most striking difference between the low anisotropy $(\mathrm{a}-\mathrm{GdAg})$ and the high anisotropy (Dy) systems is the appearance of the supplementary linear tcrm, Eq. 6, in the latter. We ascribe the origin of this term to a freezing mechanism associated with the large anisotropy. The origin of freezing is probably similar to the one observed in noncolinear, low symmetry, highly anisotropic crystalline rare earth alloys. [22] Here magnetic domains are formed by the competition between exchange and anisotropy, rather than by the usual dipolar energies. The existence of such domains in the unmagnetized state has also been discussed by Coey et al. [7] In fact, for $T<<T_{R}$, the physical arguments of Imry and Ma [23] lead to the conclusion that the domain size, L, (therefore, the spin-spin correlation length) is severely truncated by the anisotropy, i.e. $L \propto(J / D)^{2}$ a, where $a$ is of order the nearest spin neighbor distance. [24] More quantitative estimates of $\chi_{i 0}^{-1}$ require the calculation of a spin-flip field analogous to the development for crystalline alloys. [22]

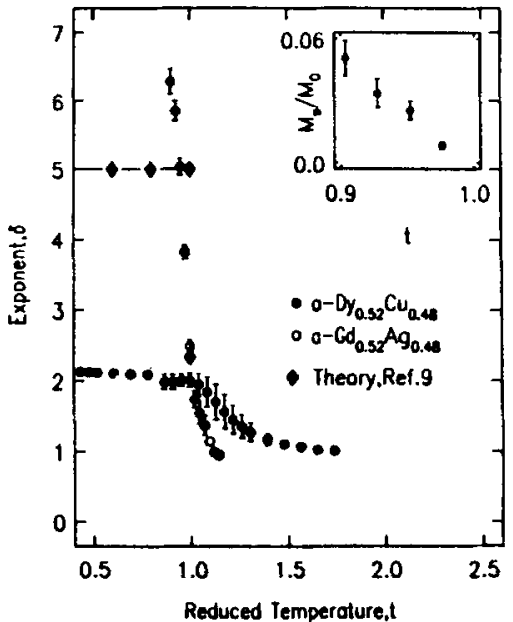

Fig. $14 \delta$, the critical magnetization exponent, $\mathrm{H} \propto \mathbf{M}^{\delta}$ as a function of reduced temperature, $t=T / T_{R}$. The insert is the "spontaneous magnetization" of a- $\mathrm{Gd}_{52} \mathrm{Ag}_{48}$ below $\mathrm{T}_{\mathrm{R}}$ expressed as the fraction of magnetization of an equivalent colinear ferromagnet.

Referring again to Fig. 14, we now compare the variation of the experimental $\delta$ between the two systems. We define $t=T / T_{R}$. The theory, solid diamonds, is meant only as a guide to the eye which indicates that large changes in $\delta$ are expected near $T_{R}$. As has been pointed out by Pytte [25] direct comparison with experimental magnitudes is unjustified, since fluctuation effects have not been included in the theory. For a-DyCu we find a rapid increase in $\delta$ near, but above $T_{\mathrm{R}}$, which saturates at $\mathrm{T}_{\mathrm{R}}(\delta=2 \pm .1)$. Apparently the freezing mechanism which prevents the first order susceptibility from increasing below $\mathrm{T}_{\mathrm{R}}$ also influences this higher order term. The behavior of the low anisotropy system a- $\mathrm{GdAg}$ (open circles) behaves in the opposite way. $\delta$ increases to very large values, suggesting the appearance of a spontaneous magnetization below $T_{R}$, although $M^{2}$ vs $H / M$ shows no clear intercept. To emphasize the appearance of a spontaneous magnetization, we refer to this transition as $T_{c}$ in Table $I$. This apparent contradiction raises the question of the size of the demagnetizing field in this thin film geometry with in-plane applied field. A standard calculation [26], assuming the limiting case of an ellipsoid of revolution with major axis $=6.5 \times 10^{-5} \mathrm{~cm}$ and minor axis $\equiv 5 \times 10^{-1} \mathrm{~cm}$, yields a demagnetizing factor $\mathrm{N}=8.2 \times 10^{-4}$. For $\mathrm{GdAg}$ with $\mathrm{M}(\mathrm{H}=5.6 \mathrm{Oe}$, $t=.9) \approx 135$ Gauss the demagnetizing field is $N M \sim .11$ Oe. Using this rough calculation we are then able to estimate the spontaneous magnetization, $M_{s}(t)$, from extrapolations of $\log$-log plots evaluated at $\mathbf{H}$ - NM. The ratio $\mathbf{M}_{\mathbf{s}}(\mathrm{t}) / \mathbf{M}_{0}$, where $\mathbf{M}_{0}$ is the expected ferromagnetic saturation value, is shown in the insert of Fig. 14. It rises rapidly below $t=1$, but has an absolute value which is only a small fraction of that for a colinear ferromagnet containing the same number of spins. At $4.2 \mathrm{~K}$ we find $\mathrm{M}_{\mathrm{s}} / \mathrm{M}_{\mathrm{o}}=.18$. The foregoing analysis does 
not depend critically on the value assumed for the demagnetizing field because of the very high $\delta$ value.

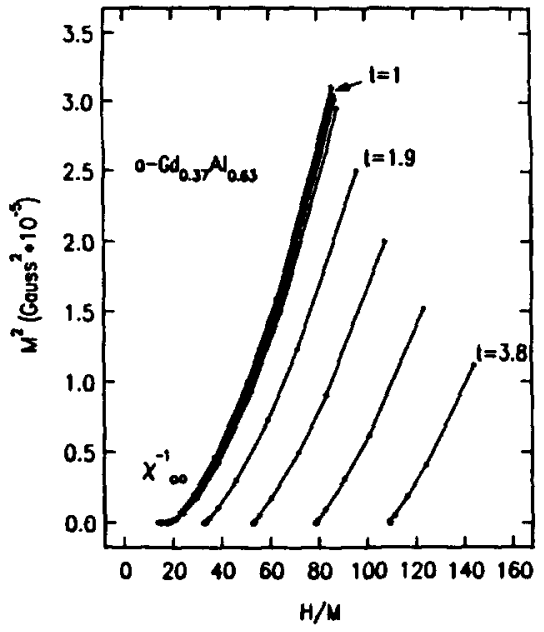

Fig. 15 Arrott $\left(\mathrm{M}^{2}\right.$ vs. $\left.\mathrm{H} / \mathrm{M}\right)$ plots of $\mathrm{a}-\mathrm{GdAl}_{2}$ for applied fields up to $48 \mathrm{kOe} . \quad \mathrm{t}=\mathrm{T} / \mathrm{T}_{\mathrm{sg}}$, where the spin glass freezing temperatures $T_{\mathrm{sg}}=15.75 \mathrm{~K}[20]$.

Finally, in Fig. 15, we show the results of our measurements on the spin glass a-Gd ${ }_{37} \mathbf{A l}_{63}[19,20]$. The differences between the spin glass and the earlier figures is apparent. Besides the obvious difference in limiting susceptibility there are more subtle variations in the curvature of the plots. For example, the curvature remains positive for all temperatures in Fig. 15, whereas changes in sign occur as a function of both $\mathrm{t}$ and $\mathrm{H}$ in Figs. 10 through 13. In order to compare experiments we have remained consistent with the earlier analyses by plotting $\log M$ vs. $\log H_{\text {eff }}$, where $H_{\text {eff }}$ in the present case is $\mathrm{H}_{\text {applied }}-13.7 \mathrm{M}$. For a more realistic analysis based on theoretical considerations using scaling arguments, see the work of Malozemoff et al. [20]. Table II summarizes our results for $\delta$ in the various systems.

Table II

$\begin{array}{lccccc} & \mathrm{T}_{\mathrm{R}} & \delta\left(\mathrm{T}<<\mathrm{T}_{\mathrm{R}}\right) & \delta\left(\mathrm{T}=\mathrm{T}_{\mathrm{R}}\right) & \delta\left(\mathrm{T}>>\mathrm{T}_{\mathrm{R}}\right) & \text { Ref. } \\ \text { a-Dy }_{.51} \mathrm{Cu}_{.48} & 23 & 2.1 \pm .1 & 2.0 \pm .1 & 1 \pm .05 & \\ \mathrm{a}-\mathrm{Dy}_{.31} \mathrm{Ni}_{.68} & 9.5 & >3.5 & & 1 \pm .05 & \\ \text { a-Gd }_{.52} \mathrm{Ag}_{.48} & 83 & >5 & 2.5 \pm .5 & 1 \pm .05 & \\ \text { a-Gd }_{.37} \mathrm{Al}_{.63} & 15.75 & 1.1 \pm .1 & 1.5 \pm .1 & 1 \pm .05 & {[20]} \\ \text { Theory } & & 5 & 2.3 & & {[9]}\end{array}$

CONCLUSIONS

Concerning the amorphous Dy alloys we conclude from the thermal data that these materials are highly anisotropic magnets in which $\mathrm{D} / \mathrm{J}$ is much greater than 1 . The large linear term also implies that the anisotropy axes are random. We find, according to an Arrott plot analysis, that none of the Dy alloys studied develop a spontaneous moment at any temperature. This is true even in matrices where equivalent concentrations of $\mathrm{Gd}$ lead to long range magnetic order. We also observe a change in the exponent $\delta,\left(H \propto M^{\delta}\right)$ near $T_{R}$. In this respect our data agree with the theoretical predictions [9].

Contrary to theory [9], however, the Dy alloys never develop an infinite susceptibility. This disagreement with theory near the origin of the Arrott plots are probably due to the fact that the theory was developed for $D / J<1$, i.e. only the leading term in $D / J$ is included in the magnetic equation of state. Furthermore, the $T=0$ arguments by Imry and $\mathrm{Ma}$ [23] suggest that the domain size is severely reduced by anisotropy and, therefore, would limit the susceptibility.

Conclusions concerning $\mathrm{Gd}$ alloys, specifically a-Gd ${ }_{52} \mathrm{Ag}_{.48}$ are more elusive. A difficulty stems from the fact that the major source and the extent of randomness of anisotropy are not known. After all, a-Gd ${ }_{37} \mathrm{Al}_{.63}$ is a spin glass. Furthermore, magnetic behavior similar to that of a-Gd ${ }_{52} \mathrm{Ag}_{48}$ has been observed by Coey et al. [27] in $a-Y_{1-x} F_{x}$ near the ferromagnet spin-glass critical concentration. We had suggested earlier that the cause of the small magnetization in a-Gd ${ }_{52} \mathrm{Ag}_{.48}$ might be an (unspecified) nonrandom component to the anisotropy [28]. Clearly random $J$ with sizeable negative component would also make comparison with the Aharony-Pytte theory inappropriate.

\section{ACKNOWLEDGEMENTS}

We are grateful to $\mathrm{H}$. Lilienthal, $\mathbf{R}$. Ruf, and A. Torressen for their technical assistance and to F. Cardone for providing the chemical analysis. We also wish to acknowledge many instructive discussions with A. Aharony, G. Grinstein, J. Janak, and E. Pytte during the course of these experiments.

\section{REFERENCES}

[1] R. W. Cochrane, R. Harris, and M. J. Zuckermann, Phys. Rep. 48, 1 (1979); this work is a comprehensive review of the early experimental and theoretical work.

[2] R. Harris, M. Plischke and M. J. Zuckermann, Phys. Rev. Lett. 31, 160 (1973).

[3] R. W. Cochrane, R. Harris, and M. Plischke, J. Noncrystalline Solids 15, 239 (1974).

[4] G. Czjzek, J. Fink, F. Goetz, H. Schmidt, J. M. D. Coey, J.-P. Rebouillat, and A. Lienard, Phys. Rev, B23, 2513 (1981).

[5] J. M. D. Coey and S. von Molnar, J. de Phys. Let. 39, L-327 (1978).

[6] S. von Molnar, C. N. Guy, R. J. Gambino, and T. R. McGuire, J. Magn. Magn. Mat. 15-18, 1391 (1980)

[7] J. M. D. Coey, T. R. McGuire, and B. Tissier, Phys. Rev, B24, 1261 (1981).

[8] S. von Molnar, B. Barbara, T. R. McGuire and R. J. Gambino, JAP 53, 1350 (1982).

[9] A. Aharony and E. Pytte, Phys. Rev. Lett. 45, 1583 (1980).

[10] S. J. Poon and J. Durand, Phys. Rev. B16, 316 (1977).

[11] R. J. Gambino and T. R. McGuire, J. Appl. Phys. 52, 2190 (1981).

[12] T. R. McGuire and R. J. Gambino, IEEE Trans. MAG14, 838 (1978).

[13] R. Asomoza, I. A. Campbell, A Fert, A. Lienard and J. P. Rebouillat, J. Phys. F9, 349 (1979).

[14] S. von Molnar, R. J. Gambino, and J. M. D. Coey, JAP 52, $2193(1981)$

[15] See e.g. B. D. Cullity, "Elements of X-Ray Diffraction," Addison-Wesley Publishing Company, Inc., Reading, Mass., 1956.

[16] E. M. Forgan and S. Nedjat, Rev. Sci. Instr. 51, 411 (1980).

[17] I. Ya Korenblit and E. F. Shender, J. Phys. F8, L-197 (1978).

[18] B. Boucher, J. de Phys. Let. 37, L-345 (1976).

[19] T. Mizoguchi, T. R. McGuire, S. Kirkpatrick, and R. J. Gambino, Phys. Rev. Lett. 38, 89 (1977).

[20] A. P. Malozemoff, B. Barbara, and Y. Imry, JAP 53, 2205 (1982); See also A. P. Malozemoff, this conference.

[21] See e.g. J. S. Kouvel and M. E. Fisher, Phys. Rev. 136, A1626 (1964); or for amorphous ferromagnets, P. Gaunt, S. C. Ho, G. Williams and R. W. Cochrane, Phys. Rev. B35, 1399 (1981).

[22] B. Barbara, Thesis, University of Grenoble (1972) and references cited therein.

[23] Y. Imry and S. Ma, Phys. Rev. Lett. 35, 1399 (1975).

[24] Although this formula is appropriate for $D / J \leqslant 1$, it demonstrates the sensitive dependence of domain size on anisotropy.

[25] E. Pytte,private communication.

[26] See e.g. A. H. Morrish in "The Physical Principles of Magnetism," John Wiley \& Sons, Inc., New York (1965), p. 10.

[27]. J. M. D. Coey, D. Givord, A. Lienard, and J. P. Rebouillat, J. Phys. F. 11, 2707 (1981).

[28] As discussed in ref. 9, the inclusion of symmetry-breaking terms leads to spontaneous magnetization. For more detailed discussion, see: A. Aharony, Solid State Comm. 28, 667 (1978); and D. Mukamel and J. Grinstein, Phys. Rev. B25, 381 (1982). 